\title{
Recidiva local y afectación metastásica única de tumor renal. Factores pronósticos y supervivencia
}

\author{
J. López Ferrandis, J. Rioja Zuazu, A. Saiz Sansi, J.Ma Regojo Balboa, \\ J.M. Fernández Montero, D. Rosell Costa
}

Servicio de Urología. Clínica Universitaria de Navarra. Pamplona.

Actas Urol Esp 2005; 29 (3): 269-275

\section{RESUMEN}

RECIDIVA LOCAL Y AFECTACIÓN METASTÁSICA ÚNICA DE TUMOR RENAL. FACTORES PRONÓSTICOS Y SUPERVIVENCIA

Objetivo: Valorar las características clínicas y patológicas y la supervivencia de los tumores renales que presentaron recidiva local o metástasis única y fueron tratadas quirúrgicamente.

Material y método: Estudio retrospectivo de 321 nefrectomías valorando las variables clínicas y patológicas de aquellos pacientes que presentaron recidiva local o metástasis única y fueron tratados quirúrgicamente. Estudio y comparación de la supervivencia en los diferentes grupos.

Resultados: El único factor influyente de forma independiente en la presentación de recidiva local es el estadio patológico. La recidiva local y la presencia de metástasis única tienen una supervivencia similar, estadísticamente peor que el resto de pacientes no metastásicos al diagnóstico, pero mejor que los pacientes con presencia de metástasis al diagnóstico.

Conclusiones: La presencia de recidiva local tiene el mismo pronóstico que la progresión en forma de una única metástasis resecable, pero mejor pronóstico que los pacientes inicialmente metastásicos a los que se realiza nefrectomía previa al tratamiento sistémico.

Palabras clave: Recidiva local. Tumor renal. Supervivencia.

\section{ABSTRACT}

LOCAL RELAPSE AND SINGLE SITE OF METASTATIC INVOLVEMENT OF RENAL TUMOUR. PROGNOSTIC FACTORS AND SURVIVAL

Objective: To evaluate the clinical and pathological characteristics and survival in patients surgically treated for renal tumours that had local recurrence or metastasis to a single site.

Material and Methods: A retrospective study of 321 nephrectomies, evaluating the clinical and pathological variables in patients having local recurrence or metastasis to a single site, and who were treated surgically. Study and comparison of survival in the different groups.

Results: The only factor found to have an independent influence on local recurrence is pathological stage. Local recurrence and the presence of metastasis to a single site have similar survival rates, both being statistically worse than in patients without metastasis at diagnosis, but better than in those having metastasis at diagnosis.

Conclusions: The presence of local recurrence has the same prognosis as a single excisable metastatic site, the prognosis being better than those initially with metastasis subjected to nephrectomy before receiving systemic treatment. 
$\mathrm{E}^{1}$ carcinoma renal (CR) presenta una incidencia de 4-6 casos / 100.000 habitantes, representando más del 90\% de los tumores malignos del parénquima renal y en la mayoría de los casos tiene su origen en las células del túbulo contorneado proximal ${ }^{1}$.

El cáncer renal representa el $2-3 \%$ de todos los tumores malignos del adulto. La incidencia y mortalidad del CR parece estar en aumento ${ }^{2}$, con una supervivencia global que oscila entre el 50$70 \%$ a los 5 años, siendo esta supervivencia dependiente del estadio, del tipo histológico y del grado nuclear ${ }^{1}$.

La cirugía radical es el único tratamiento eficaz, siempre que se reseque el tumor con un margen suficiente $^{3}$. Desafortunadamente y a pesar de un tratamiento quirúrgico agresivo un 30\% (hasta un $40 \%$ para algunos autores ${ }^{4}$ ) de los pacientes sin evidencia de metástasis en el momento de la cirugía, desarrollaran metástasis, con una mediana hasta la progresión de 1,3 años ${ }^{2}$.

\section{MATERIAL Y MÉTODOS}

De las 321 nefrectomías realizadas en nuestro centro entre enero de 1990 y diciembre de 2002, 47 presentaban metástasis en el momento del diagnóstico a los que se realizó nefrectomía previa al tratamiento inmunoterápico. En 274 pacientes no se evidenciaban metástasis en el momento de la cirugía.

De los 274 pacientes MO, durante el seguimiento se pierden 13 pacientes, 212 se encuentran libres de enfermedad y 49 presentan recidiva o progresión de la enfermedad.

De los 49 pacientes que presentaron recidiva progresión de la enfermedad, se estudian las variables clínicas y patológicas de 12 pacientes que presentaron recidiva local y de 14 pacientes que pre-

Tabla 1 sentaron una metástasis única en algún órgano de la economía a los que se les realizó exéresis quirúrgica de la lesión metástatica única. (Fig. 1).

A los 12 pacientes con recidiva local de la enfermedad, se les había realizado nefrectomía ampliada por tumor único. Se trata de 8 hombres y 4 mujeres, con una edad media de 57 años (rango 31-78). En todos ellos se realizó nefrectomía ampliada. Los resultados del estudio anatomo-patológico de las piezas quirúrgicas se muestra en la Tabla 1.

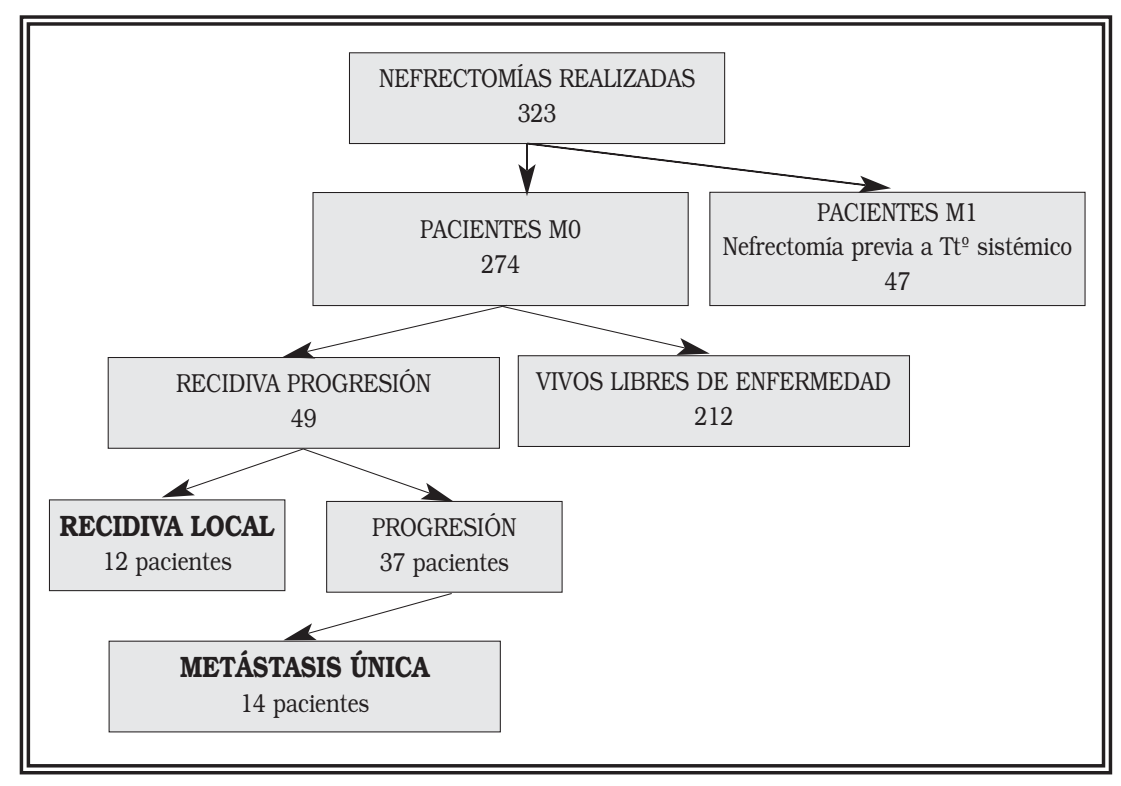

FIGURA 1. Seguimiento de las nefrectomias realizadas.

Variables patológicas de la nefrectomía en los pacientes que posteriormente presentaron recidiva local de la enfermedad

\begin{tabular}{|c|c|}
\hline Tipo histológico & $\begin{array}{l}\text { Células claras }-10 \\
\text { Carcinoma papilar }-1 \\
\text { Carcinoma sarcomatoide }-1\end{array}$ \\
\hline Tamaño & $\begin{array}{l}\text { Menor } 4 \mathrm{~cm}-3 \\
\text { Mayor } 4 \mathrm{~cm}-9\end{array}$ \\
\hline Estadio patológico & $\begin{array}{l}\text { pT2 - } 2 \\
\text { pT3 - 9 } \\
\text { pT4 - } 1\end{array}$ \\
\hline Grado de diferenciación & $\begin{array}{l}\mathrm{G} 1-1 \\
\mathrm{G} 2-2 \\
\mathrm{G} 3-5 \\
\mathrm{G} 4-1 \\
\mathrm{Gx}-3\end{array}$ \\
\hline Afectación patológica ganglionar & $\begin{array}{l}\mathrm{N} 0-6 \\
\mathrm{~N} 1-2 \\
\mathrm{~N} 2-2 \\
\mathrm{Nx}-2\end{array}$ \\
\hline
\end{tabular}


El tiempo desde la nefrectomía hasta la recidiva presentó una media de 19,6 meses, con una mediana de 11,1 meses.

Durante el seguimiento fallecieron 8 pacientes $(66,6 \%)$ con un tiempo medio desde la recidiva hasta la muerte de 28,6 meses y una mediana de 27,2 meses.

En los 14 pacientes que presentaron metástasis única durante la evolución de su enfermedad que fue tratada quirúrgicamente, la localización de las metástasis fue en pulmón en 6 pacientes, SNC en 2 pacientes, riñón o suprarrenal contralateral en 4 pacientes, región frontotemporal e hígado en 1 paciente.

El tiempo desde la nefrectomía hasta la progresión fue de 31,9 meses con una mediana de 11,1 meses. Fallecieron durante el seguimiento 9 pacientes $(64,2 \%)$, con un tiempo medio desde la progresión hasta la muerte de 24,4 meses y una mediana de 16,6 meses.

Se realiza estudio estadístico mediante el programa informático SPSS.

Se compara el grupo de pacientes con recidiva local frente a los que presentaron metástasis única y frente al resto de pacientes (pacientes MO en el momento del diagnóstico y no presentan recidiva local ni metástasis única, es decir aquellos que en su evolución no progresan o si lo hacen presentan metástasis en número de 2 o más), valorando sus diferencias y sus semejanzas. Posteriormente se compara la supervivencia entre el grupo con recidiva local de la enfermedad con el grupo que presentó metástasis única durante la evolución de la enfermedad y con el grupo que presentaba metástasis en el momento del diagnóstico.

\section{RESULTADOS}

1. Comparación del grupo con recidiva local frente al grupo de pacientes que no presentaron recidiva local ni progresión metastásica única de la enfermedad.

No existen diferencias en cuanto al sexo, el tamaño tumoral (menor de $4 \mathrm{~cm}$ vs mayor de 4 $\mathrm{cm}$ ), la lateralidad ni el tipo histológico (células claras vs resto).

Existen diferencias estadísticamente significativas respecto al estadio patológico (pT1-2 vs pT3-4) $(\mathrm{p}<0,0001)$, el grado de diferenciación (G1-2 vs G3-4) $(\mathrm{p}=0,01)$ y la afectación patológica ganglionar $(\mathrm{p}=0,01)$.

Los pacientes con recidiva local presentan peor grado histológico, peor estadio patológico y mayor afectación patológica ganglionar.

El estudio multivariado sólo muestra como factor de influencia independiente en la recidiva local de la enfermedad el estadio patológico $\mathrm{OR}=6,5 ; 95 \%$ IC $\operatorname{Exp} \mathrm{B}(1,3-32,1) \mathrm{p}=0,02$.

2. Comparación del grupo de pacientes con recidiva local frente al grupo de pacientes que presentaron metástasis única.

No se encuentran diferencias estadísticamente significativas en el tamaño tumoral, la afectación patológica ganglionar, el tipo histológico, el estadio patológico tumoral ni el grado de diferenciación.

\section{Estudio de supervivencia.}

Se compara la supervivencia de 3 grupos: el grupo de pacientes con recidiva local de la enfermedad, el grupo de pacientes que presentan metástasis única tratada quirúrgicamente y el resto de los pacientes MO en el momento del diagnóstico.

La supervivencia de los pacientes con recidiva local no presenta diferencias estadísticamente significativas $(p=0,49)$ frente al grupo de pacientes con metástasis únicas.

Por otra parte, éstos dos grupos si muestran diferencias estadísticamente significativas con el grupo de pacientes sin recidiva ni progresión única. $\mathrm{p}=0,09$ para el grupo con metástasis única y $\mathrm{p}=0,002$ para el grupo con recidiva local (Fig. 2).

Dado que los pacientes que presentaron recidiva local como los pacientes intervenidos por metástasis única presentaban una supervivencia similar, se agruparon ambos grupos para compararlos con los pacientes que presentaron metástasis en el momento del diagnóstico y se realizó nefrectomía previa al tratamiento inmunoterápico, mostrando los siguientes resultados: Existen diferencias estadísticamente significativas entre ambos grupos ( $p=0,03$; Log Rango 4,68) con una mediana de supervivencia de 21,3 y 13,8 meses respectivamente (Fig. 3). 


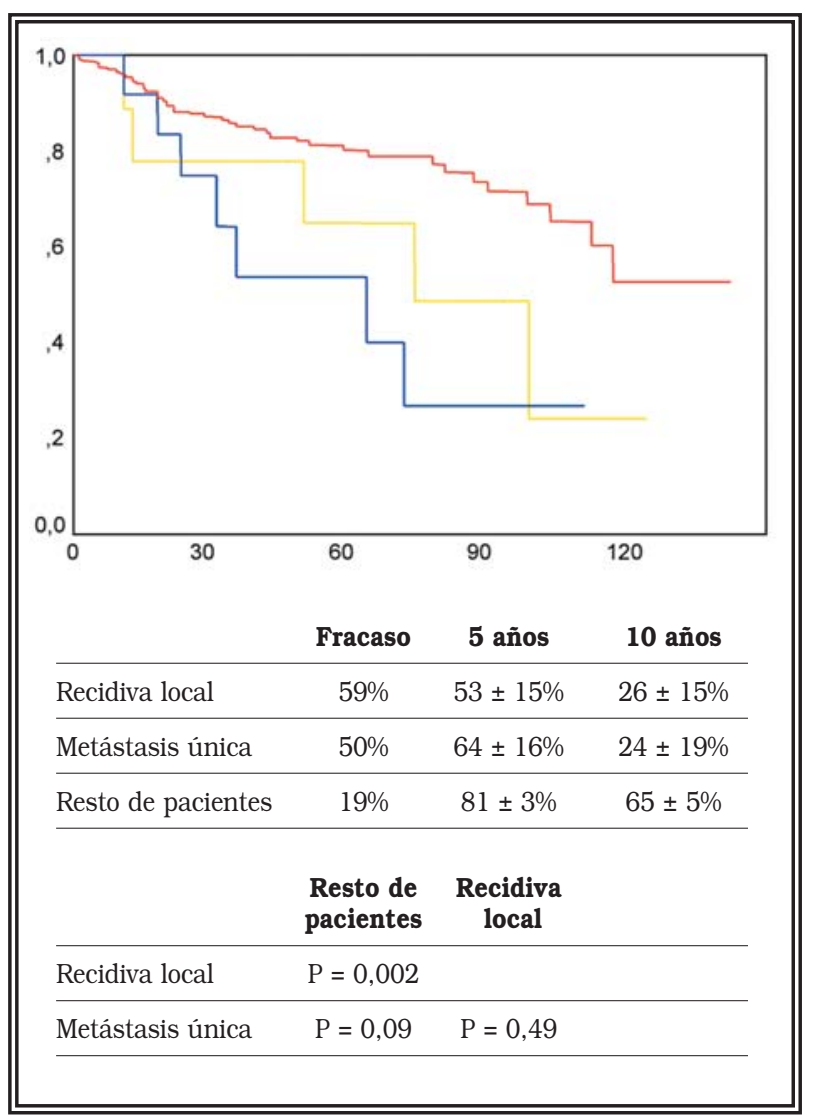

FIGURA 2. Curvas de supervivencia. Pacientes MO en el momento del diagnóstico. Recidiva local intervenida us metástasis única intervenida vs resto de pacientes. (Abscisas: porcentaje de supervivencia acumulada; ordenadas: tiempo en meses).

Los pacientes que presentan recidiva local de CR tras la nefrectomía, a pesar del tratamiento quirúrgico, presentan:

- Peor supervivencia que el grupo de pacientes MO inicialmente que no tiene recidiva local o progresión única.

- Supervivencia similar a aquellos pacientes que presentan una metástasis única y son intervenidos quirúrgicamente.

- Mejor supervivencia que los pacientes con metástasis en el momento del diagnostico a los que se trata mediante nefrectomía citorreductora e inmunoterapia.

\section{COMENTARIOS}

A pesar del diagnóstico precoz creciente, entre un $20-30 \%$ de los pacientes presentan metástasis en el momento del diagnóstico ${ }^{4}$, y supuestamente hasta un $50 \%$ son portadores de micrometástasis

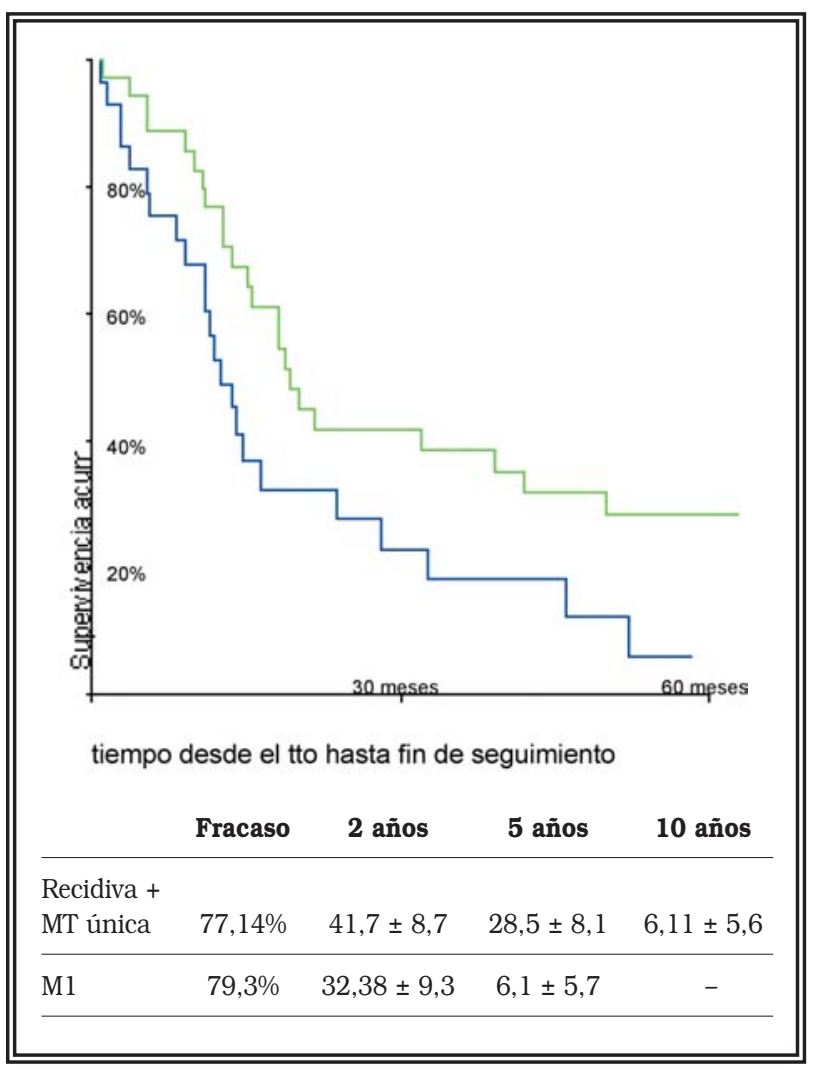

FIGURA 3. Curvas de supervivencia pacientes M1 frente a pacientes MO que presentan en la evolución recidiva local o metástasis única y son tratados con cirugía.

que pueden dar expresión clínica después del tratamiento quirúrgico ${ }^{1}$. Aproximadamente el $5 \%$ de los pacientes tratados por CR localizado presentarán recurrencia local de la enfermedad y el 25\% desarrollarán metástasis a distancia ${ }^{5}$. $\mathrm{El}$ asiento de las metástasis se localiza en un $70 \%$ de los casos en un solo órgano ${ }^{1}$. Las localizaciones más frecuentes de las metástasis son, los pulmones (50-60\%), ganglios linfáticos, huesos (25-50\%) e hígado, aunque se han descrito localizaciones en prácticamente todos los órganos del cuerpo.

Las metástasis se presentan en un $80 \%$ (hasta un $90 \%$ para algunos autores ${ }^{5}$ ) en los 3 primeros $a_{n ̃ o s}{ }^{4}$, con una media hasta el diagnóstico de 15 meses, tardando más aquellos tumores con un estadio menor. Sólo el 7\% de las metástasis ocurren más tarde de los 5 años.

La mayoría de las recurrencias para pacientes con enfermedad sin afectación patológica ganglionar aparece dentro de los 6 años tras la resección inicial, con un tiempo medio de recurrencia de 2229 meses dependiendo del estadio tumoral ${ }^{5}$. 
Para Saidi et al. ${ }^{5}$, en pacientes sin afectación ganglionar, para detectar progresión de forma temprana, se debe realizar de rutina radiografía de tórax y TC cada 6 meses durante los primeros 3 años y posteriormente 1 vez al año. Para Zisman et al. ${ }^{6}$ en los protocolos de seguimiento en los pacientes no metastásicos, se debe enfatizar más en la búsqueda de metástasis a distancia que en la búsqueda de recidiva local.

La cirugía radical es el único tratamiento efi$\mathrm{caz}^{4,7}$ por la baja quimio y radio-sensibilidad de éstos tumores. El tratamiento estándar para el $\mathrm{CR}$ es, hoy en día, la nefrectomía radical o ampliada que incluye la fascia de Gerota y su contenido aunque el beneficio frente a la nefrectomía simple no está claramente demostrado. La necesidad de añadir una linfadenectomía regional es también discutida y no aclarada. Hay autores que le atribuyen aumento de la supervivencia, pero en su contra se presentan los siguientes argumentos: metastatizan con la misma frecuencia por vía hemática que linfática, no es infrecuente encontrar metástasis a distancia sin afectación ganglionar y el drenaje linfático es variable, pudiendo drenar en cualquier ganglio retroperitoneal ${ }^{3}$.

Las técnicas de nefrectomía parcial desarrolladas en pacientes con riñones únicos, han demostrado resultados comparables en tiempo con la nefrectomía radical, lo que ha llevado a la utilización de la nefrectomía parcial como técnica electiva en pacientes con riñones contralaterales sanos y tumores menores de $4 \mathrm{~cm}$. En estos casos, con más de 4 años de seguimiento la literatura muestra un porcentaje de recidivas de un $4 \%{ }^{3}$.

$\mathrm{El}$ pronóstico de los $\mathrm{CR}$ va a depender fundamentalmente de la presencia o no de metástasis en el momento del diagnóstico, así como de las características clínicas y patológicas del tumor. Los factores pronósticos más importantes son la presencia de metástasis en el momento del diagnóstico, el estadio patológico, el grado tumoral, la variedad anatomo-patológica y el estado general previo a la nefrectomía ${ }^{8}$.

En una revisión realizada por nuestro grupo, tras realizar un estudio multivariado, se mantuvieron como factores independientes influyentes en la progresión de la enfermedad el estadio patológico, la afectación ganglionar y el grado nu- clear $^{1}$. El peor factor pronóstico es la presencia de metástasis con una supervivencia de 5-10\% a 5 años y 0-7\% a 10 años (sin tratamiento, los pacientes metastásicos tienen una supervivencia media de 6-10 meses con 10-20\% de supervivencia a los 2 años). Algunos autores ${ }^{7}$ afirman que en éste grupo de pacientes la supervivencia está estrictamente relacionada con la agresividad tumoral más que con el emplazamiento de las metástasis, aunque para otros ${ }^{9}$, la localización sí es un factor pronóstico, así, la presencia de metástasis pulmonares se asocia a mejor pronóstico que las metástasis en otros lugares como el hueso, el cerebro o el hígado. Mejean et al. ${ }^{9}$ afirman que la presencia de metástasis en un solo órgano (entendiendo que en ése único órgano pueden presentarse un número múltiple de metástasis) se correlaciona con mejor supervivencia que la afectación de diferentes órganos y que la aparición de las metástasis en los primeros 12 o en los primeros 24 meses tras el diagnóstico se asocia a peor pronóstico.

Resulta evidente que el comportamiento biológico del $\mathrm{CR}$ resulta de complejas interacciones entre múltiples factores pronósticos ${ }^{6}$. Se ha buscado y se sigue buscando un tratamiento eficaz para los pacientes que presentan metástasis en el momento del diagnóstico y para los que tienen recidiva local o a distancia. El tratamiento exclusivo con cirugía para CR metastásicos, único tratamiento considerado en otro tiempo, ha demostrado beneficios en pacientes con metástasis únicas resecables, que desafortunadamente sólo ocurre en un 1-3\% de los pacientes con CR metastásico ${ }^{10} \mathrm{y}$, las curiosas regresiones espontáneas de las metástasis tras la exéresis del tumor primario sólo suceden de forma extraordinaria.

En los CR metastásicos la realización de nefrectomía se justifica por el beneficio que puede aportar al tratamiento con inmunoterapia adoptiva, aunque la controversia está en cual de los tratamientos debe realizarse primero y a que pacientes. Los partidarios de la nefrectomía previa al tratamiento inmunoterápico, sugieren que la cirugia palia los síntomas locales, mejora la calidad de vida del paciente y probablemente mejora la respuesta al tratamiento inmunoterápico y la supervivencia, mientras que los detractores 
afirman que la morbilidad asociada a la cirugía puede retrasar en un número considerable de pacientes el inicio del tratamiento sistémico ${ }^{10}$. A pesar de todo y en el caso de pacientes seleccionados (posibilidad de que la cirugia disminuya un $75 \%$ de la masa tumoral, ausencia de metástasis en SNC, hígado y hueso, adecuada función cardiovascular y respiratoria y estado general aceptable), la terapia combinada ha demostrado una media de respuesta parcial o completa del $18-21 \%$ con una media de supervivencia de 20,5 meses $^{11}$

La nefrectomía y excisión de metástasis de pequeño volumen, especialmente en pulmón, puede beneficiar a un pequeño grupo de pacientes. La resección de las metástasis pulmonares presenta una supervivencia a 5 años de $44 \%^{12}$. Cuando las metástasis son resecables, la cirugía parece ser el mejor tratamiento incluso cuando se trata de metástasis cerebrales. La posibilidad de una completa exéresis curativa del tumor parece ser un importante factor pronóstico.

Para las metástasis cerebrales tratadas Sheehan et al. ${ }^{13}$ presentan su experiencia con 69 pacientes a los que se realizó cirugía estereotáxica por metástasis cerebrales de CR y concluyen que realiza control local en $96 \%$ con una media de prolongación de la supervivencia de 15 meses. También con gamma knife se han publicado resultados similares, algunos con supervivencias de largo tiempo ${ }^{14-16}$.

Hay pocos datos de reseción de metástasis óseas, con un incremento en la supervivencia de hasta 34 meses, en estos casos, cuando la exéresis es posible, algunos consideran ésta intervención como deseable ${ }^{17}$.

Los resultados encontrados en la bibliografia con los procedimientos quirúrgicos, apenas mejoran los resultados de la progresión espontánea del CR metastásico, aunque algún autor ${ }^{18}$ se proclama a favor de la cirugía de las lesiones residuales en pacientes que responden al tratamiento sistémico con una mediana de supervivencia de 26 meses.

A pesar de todo, no debemos olvidar que el tratamiento para el CR metastásico sigue siendo paliativo y debe ser medido en términos de duración de supervivencia y mejora de la calidad de vida.
Para Fischer et al. ${ }^{12}$ la recurrencia local, condición poco frecuente, generalmente es intervenida y se puede lograr un incremento en la supervivencia en casos individuales. Wiesner et al. ${ }^{19}$ presentan el estudio realizado con 15 pacientes que presentan recurrencia local después de nefrectomía, con un tiempo medio hasta la recurrencia de 23 meses y tiempo medio hasta la progresión de 22,2 meses. La supervivencia tumor específica es de $47 \%$ al año y $33 \%$ a los 3 años y concluyen que la cirugía está recomendada en la recurrencia local del CR y no se ha encontrado beneficio para ninguna terapia adyuvante.

En nuestra serie, los pacientes intervenidos por recidiva local presentan un tiempo medio desde la recidiva hasta la muerte de 28,6 meses y mejor supervivencia que los pacientes metastásicos.

\section{CONCLUSIONES}

La recidiva local del CR muestra el mismo pronóstico que la progresión metastásica única en cualquier otro órgano de la economía.

Los pacientes con recidiva en lugar único intervenida, tanto en fosa renal como en cualquier otro órgano de la economía, muestran mejor supervivencia que los pacientes con presencia de metástasis en el momento del diagnóstico.

A nuestro juicio en aquellos pacientes con buen estado general y tumores resecables está indicada una actitud quirúrgica seguida de tratamiento inmunoterápico adyuvante.

\section{REFERENCIAS}

1. Sánchez D, López J, Arocena J, et al. Clínica, diagnóstico y pronóstico del carcinoma renal. Actas Urol Esp 2002;26(8): 532-540.

2. BC Leibovich, ML Blute, Cheville JC, et al. Prediction of progression after radical nephrectomy for patients with clear renal cell carcinoma. Cancer 2003;97(7):1663-1671.

3. Arocena J, López J, Sánchez D, et al. Tratamiento del carcinoma renal. Actas Urol Esp 2002;26(8):541-545.

4. Ljungberg B, Alamdari FI, Ramuson T, Roos G. Follow-up guidelines for nonmetastatic renal cell carcinoma based on the occurrence of metastases after radical nephrectomy. BJU International 1999;84:401-411.

5. Saidi JA, Newhouse JH, Sawczuk IS. Radiologic follow-up of patients with $\mathrm{T} 1-3 \mathrm{a}, \mathrm{b}, \mathrm{c}$ or $\mathrm{T} 4 \mathrm{~N}+\mathrm{M} 0$ renal cell carcinoma after radical nephrectomy. Urology 1998;52(6):10001003. 
6. Zisman A, Pantuck AJ, Wieder J, et al. Risk group assessment and clinical outcome algorithm to predict the natural history of patients with surgically resected renal cell carcinoma. J Clin Oncol 2002;20:4559-4566.

7. Ficarra V, Righetti R, Pilloni S, et al. Prognosis factors in patients with renal cell carcinoma: Retrospective analysis of 675 cases. European Urology 2002;41:190-198.

8. Sánchez D, Arocena J, Regojo JM, et al. Factores pronósticos en el carcinoma renal pT3. Actas Urol Esp 2003;27(1):26-32.

9. Mejean A, Ouard S, Thiounn N. Prognosis factors of renal cell carcinoma. J Urol 2003;169:821-827.

10. Wood C G. The role of cytoreductive nephrectomy in the management of metastatic renal cell carcinoma. Urol Clin N Am 2003;30:581-588.

11. Fallick ML, McDermott, David F, et al. Nephrectomy before interleukin-2 therapy for patients with metastatic renal cell carcinoma. J Urol 1997;158(5):1691-1695.

12. Fischer CG, Schmid H. Operative therapy in disease progresion and local recurrence of renal cell carcinoma. Urol Int 1999;63(1):10-15.

13. Sheehan JP, Sun MH, Kondziolka D, Flickinger J, Lunsford LD. Radiosurgery in patients with renal cell carcinoma metastasis to the brain: long-term outcomes and prognostic factors influencing survival and local tumor control. J Neurosurg 2003;98(2):342-349.

14. Mori Y, Kondziolka D, Flickinger JC, Logan T, Lunsford LD. Stereotactic radiosurgery for brain metastasis from renal cell carcinoma. Cancer 1998;83(2):344-353.
15. Simonova G, Liscak R, Novotny J Jr, Novotny J. Solitary brain metastases treated with the Leksell gamma knife: prognostic factors for patients. Radiother Oncol 2002;57 (2):207-213.

16. Schoggl A, Kitz K, Ertl A, Dieckmann K, Saringer W, Koos WT. Gamma-knife radiosurgery for brain metastases of renal cell carcinoma: results in 23 patients. Acta Neurochir 1998;140(6):549-555.

17. Kollender Y, Bickels J, Price WM, et al. Metastatic renal cell carcinoma of bone: indications and technique of surgical intervention. J Urol 2000;164:1505.

18. Sella A, Swanson DA, Ro JY et al. Surgery following response to interferon-alpha-based therapy for residual renal cell carcinoma. J Urol 1993;149:19-22.

19. Wiesner C, Jakse G, Rohde D. Therapy of local recurrence of renal cell carcinoma. Oncol Rep 2002;9(1):189-192.

Dr. J. López Ferrandis

Servicio de Urología

Clínica Univ. de Navarra

Apartado, 4209

31080 Pamplona (Navarra)

(Trabajo recibido el 14 junio de 2004) 